\title{
Factores de riesgo socio-demográficos del síndrome cardiopulmonar por hantavirus
}

\author{
Cecilia Vial C. ${ }^{1}$, Francisca Valdivieso R. ${ }^{1}$, Analía Cuiza V. , Iris Delgado B. ${ }^{2}$, Grazielle Ribeiro E' ., \\ Elena Llop R. ${ }^{3}$, Marcela Ferrés G. ${ }^{4}$, Gabriela M. Repetto L. ${ }^{5,6}$, Raúl Riquelme O. , \\ M. Luisa Rioseco Z. ${ }^{7}$, Mario Calvo A. ${ }^{8}$, Gregory Mertz $^{9}$ y Pablo A. Vial C. ${ }^{1,6}$
}

'Programa Hantavirus, Instituto Ciencias e Innovación en Medicina, Facultad de Medicina, Clínica Alemana Universidad del Desarrollo. Santiago, Chile. ${ }^{2}$ Centro de Epidemiologia en Salud y Políticas Públicas, Instituto Ciencias e Innovación en Medicina, Facultad de Medicina, Clínica Alemana Universidad de

Desarrollo. Santiago, Chile. ${ }^{3}$ Programa de Genética HumanaInstituto de Ciencias Biomédicas, Facultad de Medicina,

Universidad de Chile. Santiago,

${ }^{4}$ Departamento de Enfermedades

Infecciosas e Inmunología Pediátricas, Pontificia Universidad Católica de Chile. Santiago, Chile. ${ }^{5}$ Centro de Genética y Genómica, Instituto Ciencias e Innovación en Medicina, Facultad de Medicina, Clínica Alemana Universidad de

Desarrollo. Santiago, Chile. 'Departamento de Pediatría Clínica Alemana. Santiago, Chile. ${ }^{7}$ Facultad de Medicina, Instituto de Medicina, Universidad San Sebastián, sede Patagonia, Chile

${ }^{8}$ Facultad de Medicina

Universidad Austral de Chile (Hospital Base de Valdivia). Valdivia, Chile.

UNM Health Science Center, University of New Mexico, Albuquerque, NM, Estados

Unidos.

Los autores no tienen conflicto de interés que declarar.

Fuentes de financiamiento: National of Allergy and Infectious

Diseases at the National Institutes of Health NIH 5UI9AI045452, Fondo Nacional de Desarrollo Científico y Tecnológico FONDECYT Project No 1040155 No 11110397 y 1161447

Recibido: 11 de enero de 2019 Aceptado: 12 de junio de 2019

Correspondencia a: Cecilia Vial Cox mcvial@udd.c

\section{Introducción}

$\mathrm{E}$ 1 hantavirus tipo Andes (ANDV) es el agente etiológico del síndrome cardiopulmonar por hantavirus (SCPH) en Chile y en el sur de Argentina, con una tasa de letalidad, en promedio, de $35 \% \%^{1,2}$. La infección por ANDV tiene un período de incubación entre 10 y 40 (promedio 18) días ${ }^{3}$, seguido de una fase prodrómica de 2 a 6 días de duración, caracterizada por fiebre, mialgias, cefalea y puede incluir síntomas gastrointestinales como dolor abdominal, náuseas, vómitos y diarrea ${ }^{4.5}$. Luego, en una proporción significativa de los casos, se desarrolla una fase cardiopulmonar con insuficiencia respiratoria de comienzo agudo, asociada a disnea y tos, causado por filtración de plasma a nivel alveolar y edema pulmonar. En los casos más graves se produce una disfunción miocárdica con bajo gasto cardíaco e hipotensión arterial que requiere el uso de fármacos vasoactivos ${ }^{4,5}$. Los factores que condicionan la intensidad de la filtración de plasma, edema pulmonar y depresión miocárdica no se han establecido con precisión, habiéndose estudiado factores genéticos, inmunológicos, mediadores inflamatorios y carga viral.
El ANDV es el único hantavirus identificado en Chile y se encuentra acorde a la distribución geográfica del roedor que es su reservorio (Oligoryzomys longicaudatus), registrándose casos desde Atacama (IV ${ }^{\circ}$ Región) a Aysén (XI ${ }^{\circ}$ Región) (latitud entre $31^{\circ} 53^{\prime}$ a $\left.45^{\circ} 34^{\prime}\right)^{7}$. Análisis filogenéticos, realizados a partir de los segmentos $\mathrm{S}$ y $\mathrm{M}$ del genoma viral, identifican dos clados que concuerdan con dos eco-regiones chilenas ${ }^{8}$. Aunque no se ha estudiado si estos clados tienen relación con la gravedad del SCPH, las características epidemiológicas y clínicas de estos casos son similares en las diferentes regiones del país9.

En relación a las características socio-demográficas asociadas con la progresión de la enfermedad, en una primera caracterización epidemiológica en pacientes chilenos, se observó una mayor sobrevida en niños bajo 10 años de edad. En ese estudio se describió, por primera vez en Chile, la identificación de casos de pacientes infectados que desarrollan una enfermedad leve o asintomática ${ }^{9}$. En otro estudio de 82 pacientes chilenos bajo 15 años de edad, infectados por hantavirus, no se observó diferencias significativas de letalidad por edad de estos pacientes, ni tampoco cuando ésta se comparaba con la de adultos; en este grupo pediátrico tampoco hubo 
diferencias de letalidad por sexo ${ }^{10}$. McNeil y cols., analizaron 510 casos con SCPH por hantavirus Sin Nombre (SNV) en Estados Unidos de América (E.U.A.) entre los años 1993 y 2009, sin encontrar diferencias en la letalidad por edad, sexo, raza, etnia ni en distintas regiones geográficas ${ }^{11}$. En contraposición a estos hallazgos, el estudio de 710 casos en Argentina demostró una letalidad significativamente más alta en mujeres (34 vs 21\%), según regiones, si bien hay circulación de diferentes virus $^{12}$. Por último, estudios realizados en Paraguay, en una región endémica para el hantavirus Laguna Negra, y en el norte de Argentina, mostraron que los amerindios, tanto argentinos como paraguayos, tienen una seroprevalencia mayor para hantavirus, pero se ven menos afectados por la enfermedad en comparación con otros grupos étnicos de origen europeo que viven en el área (menonitas en el caso de Paraguay) ${ }^{13}$. Con todos estos antecedentes, nos pareció importante analizar factores socio-demográficos como modificadores de SCPH en pacientes chilenos infectados por ANDV.

\section{Pacientes y Métodos}

Este es un estudio de asociación entre características socio-demográficas y gravedad de la infección por ANDV.

\section{Pacientes}

Todos fueron enrolados entre los años 2004 y 2013, en dos protocolos de estudio de posibles factores biológicos que pudieran modificar la evolución de la enfermedad, en ocho centros de investigación clínica que eran parte de las colaboraciones del Programa Hantavirus en Chile.

\section{Criterios de inclusión}

Infección por ANDV presente (aguda) o pasada, confirmada por la detección de anticuerpos para ANDV e inmunosorbent assays (ELISAs, o RT-qPCR para ANDV) ${ }^{15}$.

\section{Definiciones}

Mortalidad: En pacientes agudos, se registró la mortalidad hasta los treinta días post-diagnóstico.

Gravedad: Para el análisis de la gravedad de la enfermedad, se definió como paciente con enfermedad grave, los que requirieron ventilación mecánica $\mathrm{y} / \mathrm{o}$ shock (hipotensión arterial y uso de fármacos vasoactivos). Enfermedad leve correspondieron a aquellos que presentaron una enfermedad caracterizada sólo por síntomas prodrómicos o que progresaron a una insuficiencia respiratoria leve, con requerimiento de oxígeno, pero sin necesidad de conexión a ventilación mecánica y que permanecieron hemodinámicamente estables, sin shock. El grupo de pacientes con enfermedad leve incluyó tres casos seropositivos para ANDV diagnosticados retrospectivamente, y que no presentaron antecedentes de enfermedad pulmonar o cardiovascular ni hospitalización por estas causas.

Etnicidad: Fue asignada a partir de los apellidos paterno y materno de los pacientes, de la siguiente manera: si por lo menos uno de los dos apellidos era de origen amerindio $^{16}$, el paciente fue reportado como tal, si los dos apellidos eran de origen europeo, se consideraron de esta etnia ${ }^{17,18}$.

Protocolo aprobado por el Comité de Ética de la Facultad de Medicina Clínica Alemana Universidad del Desarrollo y todos los participantes dieron su consentimiento informado por escrito.

\section{Análisis estadístico}

Se utilizó la prueba de $\chi^{2}$, Fisher o ANOVA para estudiar la asociación entre los factores socio-demográficos y el curso clínico. Para las variables socio-demográficas estadísticamente significativas, se implementó una regresión logística cuya variable dependiente fue gravedad de la enfermedad. Todos los análisis estadísticos se realizaron con el software IBM SPSS Statistics versión 20 (IBM). Para todas las pruebas, la significancia estadística se estableció en un valor de $\mathrm{p}<0,05$.

\section{Resultados}

El estudio incluyó 139 pacientes con infección confirmada por ANDV. La edad media de los pacientes fue de 37 años con un rango de 10-77 años; 92 pacientes eran de sexo masculino $(66,2 \%)$.

Setenta y cinco (54\%) pacientes presentaron un cuadro clínico leve, 64 pacientes desarrollaron enfermedad grave (46\%) y 12 de ellos (19\%) murieron; todos aquellos con desenlace fatal presentaron shock además de insuficiencia respiratoria con requerimiento de ventilación mecánica. Las características demográficas de los pacientes y su asociación con evolución clínica se muestran en la Tabla 1.

No se encontró una diferencia significativa en la gravedad del SCPH entre hombres y mujeres (prueba de $\chi^{2}$ de Pearson $\mathrm{p}=0,378$ ) o con la edad de los pacientes (ANOVA $\mathrm{p}=0,77$ ). Sin embargo, el SCPH grave fue significativamente mayor en personas de etnia europea (prueba de Fisher $\mathrm{p} \leq 0,05$ ).

Se implementó un modelo de regresión logística para la variable etnia, ya que ésta tiene una diferencia estadísticamente significativa entre pacientes leves y graves. El modelo de regresión logística (Tabla 2), muestra que la etnicidad europea tiene un riesgo 5,1 veces mayor de desarrollar una enfermedad grave, comparado con la amerindia $(\mathrm{OR}=5,1$; IC 95\% $=1,63-15,88)$.

Posteriormente, se evaluó la región de exposición, agrupadas en cuatro macro-regiones (Tabla 1). No se 


\begin{tabular}{|c|c|c|c|c|c|c|}
\hline \multirow[t]{2}{*}{ Variable } & & \multicolumn{2}{|c|}{ Leve } & \multicolumn{2}{|c|}{ Grave } & \multirow[t]{2}{*}{ Valor $p$} \\
\hline & & $\mathbf{n}$ & $\%$ & $\mathbf{n}$ & $\%$ & \\
\hline \multirow[t]{2}{*}{ Sexo } & Femenino & 24 & 51,1 & 23 & 48,9 & $0,378^{\mathrm{a}}$ \\
\hline & Masculino & 51 & 55,4 & 41 & 44,6 & \\
\hline \multirow[t]{2}{*}{ Etnia } & Europea & 56 & 48,3 & 60 & 51,7 & $0,002^{a}$ \\
\hline & Amerindia & 19 & 82,6 & 4 & 17,4 & \\
\hline Edad (media, DE) & & \multicolumn{2}{|c|}{$37,8(15,4)$} & \multicolumn{2}{|c|}{$37,0(14,8)$} & $0,777^{\mathrm{b}}$ \\
\hline \multirow[t]{5}{*}{ Región de exposición } & Centro-Sur (VI,VII, VIII,XIII) & 16 & 42 & 22 & 58 & $0,668^{c}$ \\
\hline & Sur 1 (IX) & 10 & 48 & 11 & 52 & \\
\hline & Sur $2(X, X I V)$ & 15 & 58 & 11 & 42 & \\
\hline & Extremo Sur (XI) & 7 & 47 & 8 & 53 & \\
\hline & Sin dato & 26 & 67 & 13 & 33 & \\
\hline \multirow[t]{3}{*}{ Tipo de residencia } & Urbano & 17 & 38,6 & 27 & 61,4 & $0,013^{a}$ \\
\hline & Rural/Marginal Urbano & 53 & 60,9 & 34 & 39,1 & \\
\hline & Sin dato & 5 & 62,5 & 3 & 37,5 & \\
\hline \multirow[t]{4}{*}{ Tipo de exposición } & Peridomiciliar & 14 & 46,7 & 16 & 53,3 & $0,258^{c}$ \\
\hline & Laboral & 26 & 57,8 & 19 & 42,2 & \\
\hline & Recreacional & 12 & 38,7 & 19 & 61,3 & \\
\hline & Sin dato & 23 & 69,7 & 10 & 30,3 & \\
\hline \multirow[t]{3}{*}{ Clasificación del lugar de exposición } & Urbano & 3 & 42,9 & 4 & 57,1 & $0,521^{\mathrm{a}}$ \\
\hline & Rural/Marginal Urbano & 49 & 49,5 & 50 & 50,5 & \\
\hline & Sin dato & 23 & 69,7 & 10 & 30,3 & \\
\hline
\end{tabular}

Tabla 2. Variables etnia y residencia en la probabilidad de desarrollar síndrome cardiopulmonar por hantavirus. Modelos de regresión logística

\begin{tabular}{lcccccc}
\hline Variable & B & Error & Valor $\mathbf{p}$ & OR & \multicolumn{2}{c}{ IC 95\% } \\
& 1,627 & 0,581 & 0,005 & 5,1 & 1,631 & 15,882 \\
Etnia & 0,9 Inf & Sup \\
Tipo de residencia & 0,907 & 0,38 & 0,017 & 2,5 & 1,176 & 5,21 \\
\hline
\end{tabular}

Tabla 3. Asociación entre las variables etnia y tipo de residencia en pacientes con síndrome cardiopulmonar por hantavirus

\begin{tabular}{llccccc}
\hline Variable & & \multicolumn{2}{c}{ Residencia urbana } & \multicolumn{2}{c}{ Residencia rural } & Valor $\mathbf{p}$ \\
& & $\mathbf{n}$ & $\mathbf{\%}$ & $\mathbf{n}$ & $\%$ & \\
\multirow{2}{*}{ Etnia } & Europea & 39 & 36,1 & 69 & 63,9 & \\
& Amerindia & 5 & 21,7 & 18 & 78,3 & $0,229^{a}$ \\
& & & & & & \\
\hline
\end{tabular}

astadístico exacto de Fisher. encontraron diferencias significativas en la gravedad del SCPH según la región de exposición $\left(\chi^{2}\right.$ de Pearson $\mathrm{p}=0,668)$.

Tampoco se encontraron diferencias significativas entre pacientes leves y graves al comparar el tipo de exposición, ya fuese peri-domiciliar, laboral o recreacional $\left(\chi^{2}\right.$ de Pearson $\left.p=0,258\right)$ o la clasificación del lugar de exposición: urbano versus rural/marginal urbano (Fisher $\mathrm{p}=0,251)$. Sin embargo, el SCPH fue significativamente mayor en individuos que vivían en un lugar urbano (Fisher $\mathrm{p} \leq 0,05)$.

En el modelo de regresión logística implementado, se observó que personas con un tipo de residencia urbana, tenían un riesgo 2,5 veces mayor que personas con un tipo de residencia rural, de desarrollar una enfermedad grave $(\mathrm{OR}=2,5 ; \mathrm{IC}=1,18-5,21)$. Para verificar si las dos variables asociadas al curso clínico de SCPH estaban asociadas entre sí, realizamos una prueba de Fisher. Se encontró (Tabla 3) que la residencia de los pacientes no está asociada a su etnia (Fisher $\mathrm{p}=0,229)$. 


\section{Discusión}

En este estudio, se analizaron los factores sociodemográficos como modificadores del curso clínico del SCPH en individuos chilenos que se infectaron con ANDV. No se encontraron diferencias significativas entre la gravedad de la enfermedad y la edad o el sexo de los pacientes, coincidiendo con lo observado en E.U.A. ${ }^{14}$ en SCPH por infección por el virus Sin Nombre y difiriendo de lo observado en Argentina, donde se ha informado una mayor letalidad en mujeres y en diferentes áreas geográficas $^{12}$. A diferencia de Argentina donde se han identificado diferentes tipos de hantavirus causando SCPH, en Chile (ANDV) y en E.U.A. (Sin Nombre virus), la información reportada en este estudio, corresponde predominantemente a infección por sólo un tipo de hantavirus ${ }^{8}$.

En nuestra serie se identificó una diferencia estadísticamente significativa para la gravedad en diferentes etnias: individuos de ascendencia europea tienen 5,1 veces más riesgo de desarrollar una enfermedad grave que los de ascendencia amerindia. Suponiendo que el hantavirus siempre ha estado presente en el sur de Chile, y que los amerindios llegaron hace más de 10.000 años, mientras que los españoles llegaron a Chile a mediados del siglo XVI, estos últimos han estado expuestos al virus por un tiempo significativamente menor. La prolongada exposición al ANDV en poblaciones nativas, podría tener un efecto evolutivo que confiera una protección biológica a la infección grave por hantavirus en la población amerindia. Una observación similar realizada en Paraguay, mostró que la prevalencia de anticuerpos contra hantavirus es mayor entre los nativos ( 17 a $40 \%$, dependiendo de la región) indicando una alta tasa de exposición; por lo contrario, la mayoría de los casos que se enferman debido a la infección por hantavirus son menonitas (ancestros europeos/ alemanes) y otras poblaciones no indígenas ${ }^{13,19}$ en los que existe una baja seroprevalencia, indicando también menor exposición al virus o mayor virulencia de la infección en esa población. Por otra parte, en las regiones endémicas de hantavirus en Paraguay, las personas de ascendencia amerindia han exhibido una enfermedad más leve que las personas con ancestros europeos, realizándose esta misma observación en una región endémica de Argentina ${ }^{13}$.

En este estudio, la etnicidad fue asignada según los apellidos del participante; este enfoque está validado y se ha demostrado que analizando marcadores genéticos (40 polimorfismos de nucleótido único) informativos de ancestria (AIMs) los individuos con un apellido mapuche tienen 58,6\% AIMs de origen nativo-americano, mientras que individuos con dos apellidos mapuches presentan un promedio de $78,45 \%{ }^{18}$. Por lo tanto, podemos suponer que los individuos que fueron clasificados como amerindios por sus apellidos son efectivamente de esa etnia. Dado que esto está basado en promedios, podrían haber individuos mal clasificados, limitante que podría resolverse mediante un estudio genético posterior que compare la etnicidad asignada genéticamente con la gravedad, especialmente considerando que la estructura genética de la población chilena ha sido objeto de investigación ${ }^{20}$.

No se encontró diferencias significativas entre el curso clínico de la enfermedad y la región de exposición, el tipo de exposición (peridomiciliar, laboral o recreacional) o la clasificación del lugar de exposición (urbano o rural). No obstante, el tipo de residencia sí se asoció con el curso clínico del SCPH: individuos que tienen una residencia urbana, tienen 2,5 veces más riesgo de tener una enfermedad grave. Una hipótesis que podría explicar esta diferencia, es la rapidez de acceso a centros de salud de mayor complejidad. Existe evidencia empírica de que los pacientes rurales tardan más en llegar y pueden morirse antes de llegar al hospital de la región, llegando a consultar a estos centros sólo los pacientes menos graves. Los pacientes incluidos en este reporte corresponden a aquellos enrolados en estudios de determinantes biológicos de gravedad de la infección; considerando que los pacientes contemplados en nuestro análisis se han identificado en centros de referencia, no se incluyó a una proporción de pacientes que fallecen antes de llegar a estos centros o no alcanzaron a cumplir con el proceso de inclusión y consentimiento. Esta será una permanente limitación de los estudios de los determinantes de gravedad y letalidad, así como de estudios de intervenciones terapéuticas, en la medida que no se presenta la oportunidad para ser incluidos en estudios. Sin embargo, la activa búsqueda de infección en pacientes con manifestaciones leves y en contactos, permite apreciar con mayor amplitud el espectro de manifestaciones clínicas y gravedad de la infección.

En este estudio, $12(19 \%)$ de 64 pacientes con evolución grave fallecieron. Esta letalidad es menor a la informada históricamente en Chile, lo que puede explicarse por tratarse de pacientes que alcanzaron a ser tratados en centros de mayor complejidad. El desarrollo de shock cardiogénico, aparece como el principal factor conducente a letalidad.

Debido a que el riesgo a tener SCPH grave lo tienen individuos de etnia europea e individuos que viven en sectores urbanos, podríamos pensar que en nuestra serie de pacientes, esta etnia está más representada en las ciudades. No obstante, no encontramos tal asociación, por lo tanto, serían dos factores de riesgo independientes.

La razón del amplio espectro clínico observado en el SCPH es desconocida, pero podría estar relacionada, tanto con el lugar de residencia, como con las diferencias en la respuesta del hospedero al virus. Por un lado, se necesita un estudio epidemiológico más detallado para dilucidar cómo el lugar de residencia podría estar influyendo en el riesgo a tener SCPH grave y para definir que no sea por la limitante de enrolamiento que tenemos en este estudio. 
Con respecto a la respuesta del hospedero, ésta puede variar entre diferentes grupos étnicos. Un enfoque de genoma completo no sesgado que compare la etnicidad asignada genéticamente versus el curso clínico del SCPH podría ser útil en la búsqueda de regiones específicas del genoma asociadas con los resultados clínicos de esta enfermedad y ayudar a entender mejor su fisiopatología.

Agradecimientos. Este trabajo fue apoyado en parte por el National Institute of Allergy and Infectious Diseases at the National Institutes of Health NIH 5UI9AI045452, Fondo Nacional de Desarrollo Científico y Tecnológico FONDECYT Project $\mathrm{N}^{\circ} 1040155, \mathrm{~N}^{\circ} 11110397$ and 1161447.

\section{Resumen}

Introducción: El síndrome cardiopulmonar por hantavirus ( $\mathrm{SCPH}$ ) es causado en Chile y en el sur de Argentina por el Andes hantavirus (ANDV), el que es endémico en esta zona. La enfermedad causada por ANDV produce un aumento de permeabilidad vascular y filtración de plasma con una alta tasa de letalidad (35\%), debido principalmente a insuficiencia respiratoria por edema pulmonar y al desarrollo en los casos graves de compromiso miocárdico, hipoperfusión y shock. Aunque se sabe que los factores socio-demográficos del hospedero pueden influir en el curso y el resultado de la enfermedad, estos no se han caracterizado previamente en la población chilena. Objetivo: Evaluar la relación entre los factores socio-demográficos y la gravedad del SCPH. Pacientes y Métodos: Período de análisis 2004-20013, pacientes atendidos en ocho centros colaboradores, diagnóstico etiológico serológico o por biología molecular, se comparan SCPH leve y grave. Se analizaron 139 pacientes chilenos, 64 (46\%) con enfermedad grave, entre los cuales 12 murieron (19\%). Resultados: La etnia europea tuvo un riesgo 5,1 veces mayor de desarrollar un $\mathrm{SCPH}$ grave que la etnia amerindia, gravedad mayor que también se asoció a una residencia urbana. Conclusiones: Se observó una asociación estadísticamente significativa entre etnia, lugar de residencia y evolución de SCPH. Se discuten hipótesis que expliquen estos hallazgos.

\section{Referencias bibliográficas}

1.- Centers for Disease Control and Prevention. Hantavirus pulmonary syndrome. Chile 1997. Morb Mortal Wkly Rep. 1997; 46: 949-51. PMID: 9338456.

2.- Figueiredo L T M, Souza W M de, Ferrés M, Enria D A. Hantaviruses and cardiopulmonary syndrome in South America. Virus Res. Elsevier B.V.; 2014; 187: 43-54. doi:10.1016/j. virusres.2014.01.015.

3.- Vial P A, Valdivieso F, Mertz G, Castillo C, Belmar E, Delgado I, et al. Incubation period of hantavirus cardiopulmonary syndrome. Emerg Infect Dis. 2006;12: 1271-3. doi:10.3201/ eid1208.051127.

4.- Hallin G W, Simpson S Q, Crowell R E, James D S, Koster F T, Mertz G J, et al. Cardiopulmonary maniclinical description of 17 patienfestations of hantavirus pulmonary syndrome. Crit Care Med. 1996; 24: $252-8$

5.- Mertz G J, Hjelle B, Crowley M, Iwamoto G, Tomicic V, Vial P A. Diagnosis and treatment of new world hantavirus infections. Curr Opin Infect Dis. 2006; 19: 437-42.

6.- Duchin J, Koster F, Peters C, Simpson G, Tempest B, Zaki S R, et al. Hantavirus Pulmonary Syndrome: a clinical description of 17 patients with a newly recognize disease. The Hantavirus Study Group. N Engl J Med. 1994; 330: 949-55.

7.- Sotomayor V, Olea A, Labraña M, Castillo $\mathrm{C}$, Ortega $\mathrm{C}$, Riquelme R, et al. Diagnóstico y manejo del síndrome cardiopulmonar por hantavirus: Chile-2007. Rev Chilena
Infectol 2009; 26: 68-86. doi:10.4067/S071610182009000100013.

8.- Medina R A, Torres-Pérez F, Galeno H, Navarrete M, Vial P A, Palma R E, et al. Ecology, genetic diversity, and phylogeographic structure of andes virus in humans and rodents in Chile. J Virol. 2009; 83: 2446-59. doi:10.1128/JVI.01057-08.

9.- Sotomayor V, Aguilera X. Epidemiología de la infección humana por hantavirus en Chile. Rev Chilena Infectol 2000;17: 220-32. doi:10.4067/ S0716-10182000000300006.

10.- Ferrés M, Sandoval C, Delgado I, Sotomayor V, Olea A, Vial PA. Hantavirosis: Caracterización clínica-epidemiológica de pacientes pediátricos en Chile. Rev Chilena Infectol 2010; 27: 52-59. doi:10.4067/S0716-10182010000100009.

11.- MacNeil A, Ksiazek T G, Rollin PE. Hantavirus pulmonary syndrome, United States, 19932009. Emerg Infect Dis. 2011; 17: 1195-201. doi:10.3201/eid1707.101306.

12.- Martínez V P, Bellomo C M, Cacace M L, Suárez P, Bogni L, Padula PJ. Hantavirus pulmonary syndrome in Argentina, 19952008. Emerg Infect Dis 2010; 16: 1853-60. doi:10.3201/eid1612.091170.

13.- Ferrer J F, Jonsson C B, Esteban E, Galligan D, Basombrio M A, Peralta-Ramos M, et al. High prevalence of hantavirus infection in Indian communities of the Paraguayan and Argentinean Gran Chaco. Am J Trop Med Hyg 1998; 59: 438-44.

14.- Padula P J, Rossi C M, Valle M O Della, Martínez P V, Colavecchia S B, Edelstein A, et al. Development and evaluation of a solidphase enzyme immunoassay based on Andes hantavirus recombinant nucleoprotein. J Med Microbiol. 2000; 49: 149-55.

15.- Vial C, Martínez-Valdebenito C, Ríos S, Martínez J, Vial PA, Ferres M, et al. Molecular method for the detection of Andes hantavirus infection: validation for clinical diagnostics. Diagn Microbiol Infect Dis 2016; 84 (1): 36-9. doi:10.1016/j.diagmicrobio.2015.07.019.

16.- Painemal Morales N. Apellidos mapuche vinculados a títulos de merced. 2011. https:// docplayer.es/20802282-Apellidos-mapuchevinculados-a-titulos-de-merced.html.

17.- Youlton R, Valenzuela C. Growth patterns in height and weight in children aged 0 to 17 years and cranial circumference in children aged 0 to 2 years from medium-high and high socioeconomic status in Santiago. Comparison with growth in children from medium-low and low status in in the Northern area of Santiago. Rev Chil Pediatría 1990; Spec No: 1-22.

18.- Fuentes M, Pulgar I, Gallo C, Bortolini M-C, Canizales-Quinteros S, Bedoya G, et al. Gene geography of Chile: regional distribution of American, European and African genetic contributions. Rev Med Chile 2014; 142: 281 9. doi:10.4067/S0034-98872014000300001.

19.- Williams R J, Bryan R T, Mills J N, Palma R E, Vera I, De Velasquez F, et al. An outbreak of hantavirus pulmonary syndrome in western Paraguay. Am J Trop Med Hyg 1997; 57: $274-$ 82.

20.- Eyheramendy S, Martínez F I, Manevy F, Vial C, Repetto G M. Genetic structure characterization of Chileans reflects historical immigration patterns. Nat Commun 2015; 6: 6472. doi:10.1038/ncomms 7472 . 